\title{
Non-Linear Neutral Differential Equations with Damping: Oscillation of Solutions
}

\author{
Saad Althobati ${ }^{1,+}$, Jehad Alzabut ${ }^{2, *,+}$ (D) and Omar Bazighifan ${ }^{3,4, *,+}$ (D) \\ 1 Department of Science and Technology, University College-Ranyah, Taif University, \\ Ranyah 21975, Saudi Arabia; Snthobaiti@tu.edu.sa \\ 2 Department of Mathematics and General Sciences, Prince Sultan University, Riyadh 11586, Saudi Arabia \\ 3 Department of Mathematics, Faculty of Education, Seiyun University, Hadhramout 50512, Yemen \\ 4 Department of Mathematics, Faculty of Science, Hadhramout University, Hadhramout 50512, Yemen \\ * Correspondence: jalzabut@psu.edu.sa (J.A.); o.bazighifan@gmail.com (O.B.) \\ + These authors contributed equally to this work.
}

Citation: Althobati, S.; Alzabut, J.; Bazighifan, O. Non-Linear Neutral Differential Equations with Damping: Oscillation of Solutions. Symmetry 2021, 13, 285. https://doi.org/ $10.3390 /$ sym 13020285

Academic Editor: Mariano Torrisi Received: 20 January 2021 Accepted: 3 February 2021 Published: 7 February 2021

Publisher's Note: MDPI stays neutral with regard to jurisdictional clai$\mathrm{ms}$ in published maps and institutional affiliations.

Copyright: $(\odot 2021$ by the authors. Licensee MDPI, Basel, Switzerland. This article is an open access article distributed under the terms and conditions of the Creative Commons Attribution (CC BY) license (https:// creativecommons.org/licenses/by/ $4.0 /)$.

\begin{abstract}
The oscillation of non-linear neutral equations contributes to many applications, such as torsional oscillations, which have been observed during earthquakes. These oscillations are generally caused by the asymmetry of the structures. The objective of this work is to establish new oscillation criteria for a class of nonlinear even-order differential equations with damping. We employ different approach based on using Riccati technique to reduce the main equation into a second order equation and then comparing with a second order equation whose oscillatory behavior is known. The new conditions complement several results in the literature. Furthermore, examining the validity of the proposed criteria has been demonstrated via particular examples.
\end{abstract}

Keywords: even-order equations; non-linear equations; oscillation; Riccati substitution; comparison technique

\section{Introduction}

In the present paper, an attempt is made to study the oscillation properties of the non-linear even-order neutral differential equations with damping

$$
\left(a(t)\left(y^{(j-1)}(t)\right)^{\alpha}\right)^{\prime}+p_{1}(t)\left(y^{(j-1)}(t)\right)^{\alpha}+p_{2}(t) x^{\alpha}(\gamma(t))=0,
$$

where $t \geq t_{0}$ and

$$
y(t):=x(t)+q(t) x(\beta(t)) .
$$

Our novel outcomes are obtained by considering the following suppositions:

$\left(L_{1}\right) a \in C^{1}\left(\left[t_{0}, \infty\right)\right), a(t)>0, a^{\prime}(t) \geq 0, q, p_{1}, p_{2} \in C\left(\left[t_{0}, \infty\right)\right), p_{1}(t)>0, p_{2}(t)>0$, $0 \leq q(t)<q_{0}<1, p_{2}$ is not identically zero for large $t, \beta \in C^{1}\left(\left[t_{0}, \infty\right)\right), \gamma \in$ $C\left(\left[t_{0}, \infty\right)\right), \beta^{\prime}(t)>0, \beta(t) \leq t$ and $\lim _{t \rightarrow \infty} \beta(t)=\lim _{t \rightarrow \infty} \gamma(t)=\infty$.

$\left(L_{2}\right)$ The following relations are satisfied

$$
\gamma(t)<\beta(t), \gamma^{\prime}(t) \geq 0,
$$

and

$$
\int_{t_{0}}^{\infty}\left(\frac{1}{a(s)} \exp \left(-\int_{t_{0}}^{s} \frac{p_{1}(y)}{a(y)} \mathrm{d} y\right)\right)^{1 / \alpha} \mathrm{d} s=\infty,
$$

where $j \geq 4$ is an even natural number and $\alpha$ is a quotient of odd natural numbers.

Non-linear neutral differential equations have been extensively utilized to mathematically model several interesting phenomena that are observed in many areas of science 
and technology such as economics, biology, fluid dynamics, physics, differential geometry, engineering,control theory, materials science, and quantum mechanics. Asymptotic properties of solutions of non-linear neutral differential equations have been the objective of many researchers. Oscillation theory, however, has gained particular attention due to its widespread applications in mechanical oscillations, earthquake structures, clinical applications, frequency measurements and harmonic oscillators, which involve symmetrical properties; see the remarkable monograph of Hale [1].

Exploring the past few years, the asymptotic behavior of non-linear neutral differential equations has become a significant research area in different disciplines. In context of oscillation theory, it has been the object of research for many academics, who have investigated this notion for non-linear neutral differential and difference equations; the reader can refer to [2-11].

In [12], Liu et al. used the integral averaging technique to establish oscillation conditions for the solutions of the equation

$$
L_{x}^{\prime}+p_{1}(t)\left|\left(x^{(j-1)}(t)\right)\right|^{p_{1}-2} x^{(j-1)}(t)+p_{2}(t)|(x(\beta(t)))|^{\theta_{1}-2} x(\beta(t))=0,
$$

where $L_{x}=a(t)\left|\left(x^{(j-1)}(t)\right)\right|^{p_{1}-2} x^{(j-1)}(t)$. On the other hand, in [13], the authors obtained oscillation criteria for equations with damping via comparing with first-order equations.

Continuing the investigation, the authors in $[14,15]$ considered equation of the form

$$
y^{(j)}(t)+p_{2}(t) x(\gamma(t))=0,
$$

and used the Riccati method to ensure that the equation is oscillatory if

$$
\liminf _{t \rightarrow \infty} \int_{\gamma(t)}^{t} \Pi(s) \mathrm{d} s>\frac{(j-1) 2^{(j-1)(j-2)}}{\mathrm{e}},
$$

and

$$
\lim \inf _{t \rightarrow \infty} \int_{\gamma(t)}^{t} \Pi(s) \mathrm{d} s>\frac{(j-1) !}{\mathrm{e}},
$$

where $\Pi(t):=\gamma^{j-1}(t)\left(1-\theta_{1}(\gamma(t))\right) p_{2}(t)$

The purpose of this paper is to improve and extend the results in $[14,15]$ and establish new oscillation criteria for Equation (1). Our approach is based on the use of Riccati substitution to reduce Equation (1) into a second order equation and then compare it with a second order equation whose oscillatory behavior is known. For examining the validity of the proposed criteria, two examples with particular values are constructed.

\section{Oscillation Conditions}

The following lemmas are essential in the sequel.

Lemma 1 ([16]). Let $x \in C^{j}\left(\left[t_{0}, \infty\right),(0, \infty)\right)$. Assume that $x^{(j)}(t)$ is of fixed sign and not identically zero on $\left[t_{0}, \infty\right)$ and that there exists $a t_{1} \geq t_{0}$ such that $x^{(j-1)}(t) x^{(j)}(t) \leq 0$ for all $t \geq t_{1}$. If $\lim _{t \rightarrow \infty} x(t) \neq 0$, then for every $\mu \in(0,1)$, there exists $t_{\mu} \geq t_{1}$ such that

$$
x(t) \geq \frac{\mu}{(j-1) !} t^{j-1}\left|x^{(j-1)}(t)\right| \text { for } t \geq t_{\mu}
$$

for every $\mu \in(0,1)$.

Lemma 2 ([17]). If $x^{(i)}(t)>0, i=0,1, \ldots, j$, and $x^{(j+1)}(t)<0$, then

$$
\frac{x(t)}{t^{j} / j !} \geq \frac{x^{\prime}(t)}{t^{j-1} /(j-1) !} .
$$


Lemma 3 ([18]). Let

Then, we have these cases:

$$
\begin{array}{ll}
\left(\mathbf{I}_{1}\right): & y(t)>0, y^{\prime}(t)>0, y^{\prime \prime}(t)>0, y^{(j-1)}(t)>0 \text { and } y^{(j)}(t)<0, \\
\left(\mathbf{I}_{2}\right): & y(t)>0, y^{(m)}(t)>0, y^{(m+1)}(t)<0 \text { for some odd integer } \\
& m \in\{1,2, \ldots, j-3\}, y^{(j-1)}(t)>0 \text { and } y^{(j)}(t)<0
\end{array}
$$

for $t \geq t_{1}$, where $t_{1} \geq t_{0}$ is sufficiently large.

For the sake of simplification, we use some notations.

$$
\begin{aligned}
& \mu_{t_{0}}(t):=\exp \left(\int_{t_{0}}^{t} \frac{\theta_{1}(y)}{a(y)} \mathrm{d} y\right) \\
& \widetilde{\mu}_{0}(t):=\left(\frac{1}{\mu_{t_{1}}(t) a(t)} \int_{t}^{\infty} p_{2}(s) \mu_{t_{1}}(s) q_{2}^{\alpha}(\gamma(s)) \mathrm{d} s\right)^{1 / \alpha} \\
& \widetilde{\mu}_{k}(t):=\int_{t}^{\infty} \widetilde{\mu}_{k-1}(s) \mathrm{d} s, k=1,2, \ldots, j-2
\end{aligned}
$$

and

$$
q_{m}(t):=\frac{1}{q\left(\beta^{-1}(t)\right)}\left(1-\frac{\left(\beta^{-1}\left(\beta^{-1}(t)\right)\right)^{m-1}}{\left(\beta^{-1}(t)\right)^{m-1} q\left(\beta^{-1}\left(\beta^{-1}(t)\right)\right)}\right), m=2, j .
$$

Lemma 4. Let (9) hold. Then

$$
\left(\mu_{t_{0}}(t) a(t)\left(y^{(j-1)}(t)\right)^{\alpha}\right)^{\prime}+\mu_{t_{0}}(t) p_{2}(t)(1-q(\gamma(t)))^{\alpha} y^{\alpha}(\gamma(t)) \leq 0, \text { for } q_{0}<1
$$

and

$$
\begin{aligned}
\left(\mu_{t_{0}}(t)\right. & \left.a(t)\left(y^{(j-1)}(t)\right)^{\alpha}\right)^{\prime} \\
& +\frac{\mu_{t_{0}} p_{2}(t)}{q^{\alpha}\left(\beta^{-1}(\gamma(t))\right)}\left(y\left(\beta^{-1}(\gamma(t))\right)-\frac{y\left(\beta^{-1}\left(\beta^{-1}(\gamma(t))\right)\right)}{q\left(\beta^{-1}\left(\beta^{-1}(\gamma(t))\right)\right)}\right)^{\alpha} \leq 0,
\end{aligned}
$$

for $t \geq t_{1}$, where $t_{1} \geq t_{0}$ is sufficiently large.

Proof. Let (9) hold. It is not difficult to see that

$$
\begin{aligned}
\frac{1}{\mu_{t_{0}}(t)} \frac{\mathrm{d}}{\mathrm{d} t} & \left(\mu_{t_{0}}(t) a(t)\left(y^{(j-1)}(t)\right)^{\alpha}\right) \\
& =\frac{1}{\mu_{t_{0}}(t)}\left(\mu_{t_{0}}(t)\left(a(t)\left(y^{(j-1)}(t)\right)^{\alpha}\right)^{\prime}+\mu_{t_{0}}^{\prime}(t) a(t)\left(y^{(j-1)}(t)\right)^{\alpha}\right) \\
& =\left(a(t)\left(y^{(j-1)}(t)\right)^{\alpha}\right)^{\prime}+\frac{\mu_{t_{0}}^{\prime}(t)}{\mu_{t_{0}}(t)} a(t)\left(y^{(j-1)}(t)\right)^{\alpha} \\
& =\left(a(t)\left(y^{(j-1)}(t)\right)^{\alpha}\right)^{\prime}+p_{1}(t)\left(y^{(j-1)}(t)\right)^{\alpha} .
\end{aligned}
$$

Since $j$ is even, by Lemma 3, $\omega(\rho)>0$ and from (2), we get that $x(t) \geq(1-q(t)) y(t)$. Thus, from (1) and (12), we conclude that (10) holds.

On the other hand, from (2), we obtain

$$
\begin{aligned}
q\left(\beta^{-1}(t)\right) x(t) & =y\left(\beta^{-1}(t)\right)-x\left(\beta^{-1}(t)\right) \\
& =y\left(\beta^{-1}(t)\right)-\left(\frac{y\left(\beta^{-1}\left(\beta^{-1}(t)\right)\right)}{q\left(\beta^{-1}\left(\beta^{-1}(t)\right)\right)}-\frac{x\left(\beta^{-1}\left(\beta^{-1}(t)\right)\right)}{q\left(\beta^{-1}\left(\beta^{-1}(t)\right)\right)}\right) \\
& \geq y\left(\beta^{-1}(t)\right)-\frac{1}{q\left(\beta^{-1}\left(\beta^{-1}(t)\right)\right)} y\left(\beta^{-1}\left(\beta^{-1}(t)\right)\right),
\end{aligned}
$$

which with (1), (12) and (13) give (11). The proof is complete. 
It is well known (see [19]) that the equation

$$
\left[r(t)\left(x^{\prime}(t)\right)^{\alpha}\right]^{\prime}+p_{2}(t) x^{\alpha}(\beta(t))=0, \quad t \geq t_{0},
$$

where $\alpha>0, r, \theta_{2} \in C\left[t_{0}, \infty\right)$, is nonoscillatory if and only if the inequality

$$
v^{\prime}(t)+\alpha r^{\frac{-1}{\alpha}}(t)(v(t))^{(1+\alpha) / \alpha}+p_{2}(t) \leq 0, \quad \text { on }[t, \infty)
$$

is satisifed.

Theorem 1. Assume that (3) and (4) hold. If

$$
\left(\frac{((j-2) !)^{\alpha} \alpha \mu_{t_{1}}(\gamma(t)) a(\gamma(t))}{\left(\epsilon_{1} \gamma^{\prime}(t) \gamma^{j-2}(t)\right)^{\alpha}}\left(x^{\prime}(t)\right)^{\alpha}\right)^{\prime}+\mu_{t_{1}}(t) q_{j}^{\alpha}(\gamma(t)) p_{2}(t) x^{\alpha}(t)=0
$$

and

$$
\frac{1}{\gamma^{\prime}(t)} x^{\prime \prime}(t)+\widetilde{\mu}_{j-3}(t) x(t)=0
$$

are oscillatory for some constant $\epsilon_{1} \in(0,1)$. Then (1) is oscillatory.

Proof. Let (9) hold. From Lemma 3, we have two possible cases $\left(\mathbf{I}_{1}\right)$ and $\left(\mathbf{I}_{2}\right)$.

Let $\left(\mathbf{I}_{1}\right)$ hold. From Lemma 2, we see $y(t) \geq \frac{1}{(j-1)} t y^{\prime}(t)$ and then $\left(t^{1-j} y(t)\right)^{\prime} \leq 0$. Thus, we obtain

$$
y\left(\beta^{-1}\left(\beta^{-1}(t)\right)\right) \leq \frac{\left(\beta^{-1}\left(\beta^{-1}(t)\right)\right)^{j-1}}{\left(\beta^{-1}(t)\right)^{j-1}} y\left(\beta^{-1}(t)\right) .
$$

By Lemma 4, we have (11). Thus, (17) gives

$$
\left(\mu_{t_{1}}(t) a(t)\left(y^{(j-1)}(t)\right)^{\alpha}\right)^{\prime}+\mu_{t_{1}}(t) p_{2}(t) q_{j}^{\alpha}(\gamma(t)) y^{\alpha}\left(\beta^{-1}(\gamma(t))\right) \leq 0 .
$$

Define

$$
w(t)=\mu_{t_{1}}(t) a(t) \frac{\left(y^{(j-1)}(t)\right)^{\alpha}}{y^{\alpha}(\gamma(t))}, w(t)>0 .
$$

Differentiating $w$ and using (18), we get

$$
\begin{aligned}
w^{\prime}(t) & \leq-\mu_{t_{1}}(t) \theta_{2}(t) q_{j}^{\alpha}(\gamma(t)) \frac{y^{\alpha}\left(\beta^{-1}(\gamma(t))\right)}{y^{\alpha}(\gamma(t))} \\
& -\frac{\mu_{t_{1}}(t) a(t)\left(y^{(j-1)}(t)\right)^{\alpha}}{y^{2 \alpha}(\gamma(t))} \alpha y^{\alpha-1}(\gamma(t)) y^{\prime}(\gamma(t)) \gamma^{\prime}(t) .
\end{aligned}
$$

From Lemma 1, we see

$$
y^{\prime}(\gamma(t)) \geq \frac{\epsilon}{(j-2) !} \gamma^{j-2}(t) y^{(j-1)}(\gamma(t)) .
$$

Since $\mu_{t_{1}}(t) a(t)\left(y^{(j-1)}(t)\right)^{\alpha}$ is decreasing, we obtain

$$
\mu_{t_{1}}(t) a(t)\left(y^{(j-1)}(t)\right)^{\alpha} \leq \mu_{t_{1}}(\gamma(t)) a(\gamma(t))\left(y^{(j-1)}(\gamma(t))\right)^{\alpha}, \text { for all } t \geq \gamma(t),
$$

which implies

$$
\frac{1}{\mu_{t_{1}}^{1 / \alpha}(\gamma(t)) a^{1 / \alpha}(\gamma(t))}\left(\mu_{t_{1}}(t) a(t)\right)^{1 / \alpha} y^{(j-1)}(t) \leq y^{(j-1)}(\gamma(t)) .
$$


From (20) and (22), we have

$$
y^{\prime}(\gamma(t)) \geq \frac{\epsilon}{(j-2) !} \frac{\gamma^{j-2}(t)}{\mu_{t_{1}}^{1 / \alpha}(\gamma(t)) a^{1 / \alpha}(\gamma(t))}\left(\mu_{t_{1}}(t) a(t)\right)^{1 / \alpha} y^{(j-1)}(t) .
$$

Since $\beta^{-1}(t)>t$ and $y^{\prime}(t)>0$, we have $y\left(\beta^{-1}(t)\right)>y(t)$ and so

$$
\frac{y\left(\beta^{-1}(\gamma(t))\right)}{y(\gamma(t))}>1
$$

By using (23) and (24) in (19), we have

$$
\begin{aligned}
w^{\prime}(t) & \leq-\mu_{t_{1}}(t) \theta_{2}(t) q_{j}^{\alpha}(\gamma(t)) \\
& -\frac{\mu_{t_{1}}(t) a(t)\left(y^{(j-1)}(t)\right)^{\alpha+1}}{y^{\alpha+1}(\gamma(t))} \alpha \frac{\epsilon \gamma^{\prime}(t) \gamma^{j-2}(t)}{(j-2) !}\left(\frac{\mu_{t_{1}}(t) a(t)}{\mu_{t_{1}}(\gamma(t)) a(\gamma(t))}\right)^{1 / \alpha} .
\end{aligned}
$$

From the definition of $w$, we have

$$
w^{\prime}(t) \leq-\mu_{t_{1}}(t) \theta_{2}(t) q_{j}^{\alpha}(\gamma(t))-\frac{\alpha \epsilon \gamma^{\prime}(t) \gamma^{j-2}(t)}{(j-2) !\left(\mu_{t_{1}}(\gamma(t)) a(\gamma(t))\right)^{1 / \alpha}} w^{(\alpha+1) / \alpha}(t),
$$

which yields,

$$
w^{\prime}(t)+\frac{\alpha \epsilon \gamma^{\prime}(t) \gamma^{j-2}(t)}{(j-2) !\left(\mu_{t_{1}}(\gamma(t)) a(\gamma(t))\right)^{1 / \alpha}} w^{(\alpha+1) / \alpha}(t)+\mu_{t_{1}}(t) q_{j}^{\alpha}(\gamma(t)) p_{2}(t) \leq 0 .
$$

Thus, we conclude that (26) is nonoscillatory for every constant $\epsilon \in(0,1)$. From [19], we see that (15) is nonoscillatory, which is a contradiction.

Let $\left(\mathbf{I}_{2}\right)$ hold. From Lemma 2, we obtain

$$
y(t) \geq t y^{\prime}(t)
$$

and then $\left(t^{-1} y(t)\right)^{\prime} \leq 0$. Hence, since $\beta^{-1}(t) \leq \beta^{-1}\left(\beta^{-1}(t)\right)$, we get

$$
y\left(\beta^{-1}\left(\beta^{-1}(t)\right)\right) \leq \frac{\beta^{-1}\left(\beta^{-1}(t)\right)}{\beta^{-1}(t)} y\left(\beta^{-1}(t)\right),
$$

which with (11) yield

$$
\left(\mu_{t_{1}}(t) a(t)\left(y^{(j-1)}(t)\right)^{\alpha}\right)^{\prime}+p_{2}(t) \mu_{t_{1}}(t) q_{2}^{\alpha}(\gamma(t)) y^{\alpha}\left(\beta^{-1}(\gamma(t))\right) \leq 0 .
$$

Integrating (29) from $t$ to $\infty$, we obtain

$$
\begin{aligned}
-y^{(j-1)}(t) & \leq-\left(\frac{1}{\mu_{t_{1}}(t) a(t)} \int_{t}^{\infty} \theta_{2}(s) \mu_{t_{1}}(s) q_{2}^{\alpha}(\gamma(s)) y^{\alpha}\left(\beta^{-1}(\gamma(s))\right) \mathrm{d} s\right)^{1 / \alpha} \\
& \leq-\widetilde{\mu}_{0}(t) y\left(\beta^{-1}(\gamma(t))\right) .
\end{aligned}
$$

Integrating this inequality $j-3$ times from $t$ to $\infty$, we get

$$
y^{\prime \prime}(t)+\widetilde{\mu}_{j-3}(t) y\left(\beta^{-1}(\gamma(t))\right) \leq 0 .
$$

Define

$$
\varphi(t)=\frac{y^{\prime}(t)}{y(\gamma(t))}, w(t)>0 .
$$


Differentiating $w$, we obtain

$$
\varphi^{\prime}(t)=\frac{y^{\prime \prime}(t)}{y(\gamma(t))}-\frac{y^{\prime}(t)}{y^{2}(\gamma(t))} y^{\prime}(\gamma(t)) \gamma^{\prime}(t)
$$

Since $y^{\prime \prime}(t)<0$, we see $y^{\prime}(\gamma(t))>y^{\prime}(t)$ for all $t \geq \gamma(t)$. Thus

$$
\varphi^{\prime}(t) \leq \frac{y^{\prime \prime}(t)}{y(\gamma(t))}-\left(\frac{y^{\prime}(t)}{y(\gamma(t))}\right)^{2} \gamma^{\prime}(t)
$$

From (30), we get

$$
\varphi^{\prime}(t) \leq-\frac{\tilde{\mu}_{j-3}(t) y\left(\beta^{-1}(\gamma(t))\right)}{y(\gamma(t))}-\left(\frac{y^{\prime}(t)}{y(\gamma(t))}\right)^{2} \gamma^{\prime}(t) .
$$

Since $\beta^{-1}(t)>t$ and $y^{\prime}(t)>0$, we have $y\left(\beta^{-1}(t)\right)>y(t)$ and so

$$
\varphi^{\prime}(t) \leq-\widetilde{\mu}_{j-3}(t)-\left(\frac{y^{\prime}(t)}{y(\gamma(t))}\right)^{2} \gamma^{\prime}(t)
$$

From the definition of $\varphi$, we have

$$
\varphi^{\prime}(t) \leq-\widetilde{\mu}_{j-3}(t)-\gamma^{\prime}(t) \varphi^{2}(t)
$$

that is,

$$
\varphi^{\prime}(t)+\gamma^{\prime}(t) \varphi^{2}(t)+\widetilde{\mu}_{j-3}(t) \leq 0 .
$$

Thus, we conclude that (33) is nonoscillatory. From [19], we see that (16) is nonoscillatory, which is a contradiction. Thus, the proof is complete.

Corollary 1. Let (4) hold. If

$$
\liminf _{t \rightarrow \infty} \int_{\gamma(t)}^{t}(1-q(\gamma(s)))^{\alpha} p_{2}(s) \frac{\mu_{t_{0}}(s)}{\mu_{t_{0}}(\gamma(s))}\left(\frac{\epsilon \gamma^{j-1}(s)}{a^{1 / \alpha}(\gamma(s))}\right)^{\alpha} \mathrm{d} s>\frac{((j-1) !)^{\alpha}}{\mathrm{e}}
$$

then (1) is oscillatory.

Corollary 2. Let (3) and (4) hold. If

$$
\liminf _{t \rightarrow \infty} \int_{\beta^{-1}(\gamma(t))}^{t} p_{2}(s) \frac{\mu_{t_{0}}(s)}{\mu_{t_{0}}\left(\beta^{-1}(\gamma(s))\right)}\left(\frac{\epsilon\left(\beta^{-1}(\gamma(s))\right)^{j-1} q_{j}(\gamma(s))}{a^{1 / \alpha}\left(\beta^{-1}(\gamma(s))\right)}\right)^{\alpha} \mathrm{d} s>\frac{((j-1) !)^{\alpha}}{\mathrm{e}}
$$

and

$$
\liminf _{t \rightarrow \infty} \int_{\beta^{-1}(\gamma(t))}^{t} \beta^{-1}(\gamma(s)) \tilde{\mu}_{j-3}(s) \mathrm{d} s>\frac{1}{\mathrm{e}^{\prime}}
$$

then (1) is oscillatory.

It is well known (see [20]) that if

$$
\int_{t_{0}}^{\infty} \frac{1}{r(t)} \mathrm{d} t=\infty, \text { and } \liminf _{t \rightarrow \infty}\left(\int_{t_{0}}^{t} \frac{1}{r(s)} \mathrm{d} s\right) \int_{t}^{\infty} \theta_{2}(s) \mathrm{d} s>\frac{1}{4}
$$

then (14) is oscillatory. 
Corollary 3. Let (4) hold and $\alpha=1$. If

$$
\begin{gathered}
\int_{t_{0}}^{\infty} \frac{\epsilon_{1} \gamma^{\prime}(t) \gamma^{j-2}(t)}{(j-2) ! \mu_{t_{1}}(\gamma(t)) a(\gamma(t))} \mathrm{d} t=\infty, \\
\liminf _{t \rightarrow \infty}\left(\int_{t_{0}}^{t} \frac{\epsilon_{1} \gamma^{\prime}(s) \gamma^{j-2}(s)}{(j-2) ! \mu_{t_{1}}(\gamma(s)) a(\gamma(s))} \mathrm{d} s\right) \int_{t}^{\infty} p_{2}(s) \mu_{t_{1}}(s) q_{j}^{\alpha}(\gamma(s)) \mathrm{d} s>\frac{1}{4}, \\
\int_{t_{0}}^{\infty} \gamma^{\prime}(t) \mathrm{d} t=\infty,
\end{gathered}
$$

and

$$
\liminf _{t \rightarrow \infty}\left(\int_{t_{0}}^{t} \int_{t_{0}}^{\infty} \gamma^{\prime}(s) \mathrm{d} s\right) \int_{t}^{\infty} \widetilde{\mu}_{j-3}(s) \mathrm{d} s>\frac{1}{4},
$$

then (1) is oscillatory.

\section{Applications}

For the sake of demonstrating the validity of the above hypotheses, this section presents some particular examples in correspondence with Equation (1).

Example 1. Consider the equation

$$
\left(x(t)+\frac{1}{2} x\left(\frac{1}{2} t\right)\right)^{(4)}+\frac{\theta_{0}}{t^{4}} x\left(\frac{9}{10} t\right)=0, t \geq 1 .
$$

Note that $\alpha=1, j=4, a(t)=1, p_{2}(t)=\theta_{0} / t^{4}, \gamma(t)=9 t / 10$ and $\beta(t)=t / 2$. Applying the conditions (7) and (8) to Equation (37), we get

\begin{tabular}{ccc}
\hline Condition & (7) & (8) \\
\hline Condition & $\theta_{0}>1839.2$ & $\theta_{0}>59.5$ \\
\hline
\end{tabular}

By Corollary 1, all solutions of (37) are oscillatory if $\theta_{0}>57.5$. From this, we conclude that our results are better than those of $[14,15]$.

Example 2. For $t \geq 1$, consider the equation

$$
\left(x(t)+\frac{1}{2} x\left(\frac{t}{3}\right)\right)^{(4)}+\frac{1}{t} y^{(3)}(t)+\frac{\theta_{0}}{t^{4}} x\left(\frac{t}{2}\right)=0,
$$

where $\theta_{0}>0$ is a constant. Let $\alpha=1, j=4, a(t)=1, p_{1}(t)=1 / t, p_{2}(t)=\theta_{0} / t^{4}, \gamma(t)=$ $t / 2, \beta^{-1}(t)=(3 / 2) t$ and $\beta(t)=t / 3$. Then

$$
\mu_{t_{0}}(t)=t, \mu_{t_{0}}(\gamma(t))=t / 2
$$

Thus, we see that

$$
\begin{aligned}
& \liminf _{t \rightarrow \infty} \int_{\gamma(t)}^{t}(1-q(\gamma(s)))^{\alpha} p_{2}(s) \frac{\mu_{t_{0}}(s)}{\mu_{t_{0}}(\gamma(s))}\left(\frac{\epsilon \gamma^{j-1}(s)}{a^{1 / \alpha}(\gamma(s))}\right)^{\alpha} \mathrm{d} s \\
= & \liminf _{t \rightarrow \infty} \int_{t / 2}^{t} \frac{\theta_{0}}{t^{4}}\left(\frac{t^{3}}{8}\right) \mathrm{d} s=\frac{\theta_{0}}{8} \ln 2 .
\end{aligned}
$$

It follows that

$$
\theta_{0}>\frac{48}{\mathrm{e} \ln 2} .
$$

Using Corollary 1, we deduce that all solutions of (38) are oscillatory if $\theta_{0}>25.5$. 


\section{Conclusions}

In this paper, we establish new oscillation criteria for a certain class of even-order non-linear differential equation with damping of the form (1). Our approach is different and obtained by using Riccati technique and comparing it with second-order equations. The new proposed conditions complement several results in the literature. Furthermore, some interesting examples are presented to examine the applicability of theoretical outcomes. Establishing oscillation criteria if $\int_{t_{0}}^{\infty}\left(\frac{1}{a(s)} \exp \left(-\int_{t_{0}}^{s} \frac{p_{1}(y)}{a(y)} \mathrm{d} y\right)\right)^{1 / \alpha} \mathrm{d} s<\infty$ could be a promising topic for future work.

Author Contributions: O.B., S.A. and J.A. conceived and designed the experiments; O.B., S.A. and J.A. performed the experiments; O.B., S.A. and J.A. analyzed the data; O.B., S.A. and J.A. contributed reagents/materials/analysis tools; O.B., S.A. and J.A. wrote the paper. All authors have read and agreed to the published version of the manuscript.

Funding: J. Alzabut would like to thank Prince Sultan university for funding this work.

Institutional Review Board Statement: Not applicable.

Informed Consent Statement: Not applicable.

Data Availability Statement: Not applicable.

Acknowledgments: The authors express their sincere thanks to the anonymous referees for their valuable comments and suggestions. J. Alzabut would like to thank Prince Sultan university for supporting this work.

Conflicts of Interest: The authors declare that they have no conflict of interest.

\section{References}

1. Hale, J.K. Theory of Functional Differential Equations; Springer: New York, NY, USA, 1977.

2. Alzabut, J.; Bohner, M.; Grace, S.R. Oscilaltion of nonlinear third-order difference equations with mixed neutral terms. Adv. Differ. Equ. 2021, 2021, 3. [CrossRef]

3. Baculikova, B.; Dzurina, J.; Graef, J.R. On the oscillation of higher-order delay differential equations. Math. Slovaca 2012, 187, 387-400. [CrossRef]

4. Bazighifan, O.; Alotaibi, H.; Mousa, A.A.A. Neutral Delay Differential Equations: Oscillation Conditions for the Solutions. Symmetry 2021, 13, 101. [CrossRef]

5. Grace, S.; Alzabut, J. Oscillation results for nonlinear second order difference equations with mixed neutral terms. Adv. Differ. Equ. 2020, 2020, 8. [CrossRef]

6. Bazifghifan, O.; Ramos, H. On the asymptotic and oscillatory behavior of the solutions of a class of higher-order differential equations with middle term. Appl. Math. Lett. 2020, 107, 106431. [CrossRef]

7. Bazighifan, O.; Ahmad, H. Asymptotic Behavior of Solutions of Even-Order Advanced Differential Equations. Math. Eng. 2020, 2020, 8041857. [CrossRef]

8. Bazighifan, O.; Grace, S.R.; Alzabut, J.; Özbekler, A. New results for oscilaltory properties of neutral differential equations with a p-Laplacian like operator. Miskolc Math. Notes 2020, 21, 631-640. [CrossRef]

9. Agarwal, R.P.; Bohner, M.; Li, T.; Zhang, C. A new approach in the study of oscillatory behavior of even-order neutral delay differential equations. Appl. Math. Comput. 2013, 225, 787-794. [CrossRef]

10. Li, T.; Rogovchenko, Y.V. Rogovchenko, Oscillation criteria for even-order neutral differential equations. Appl. Math. Lett. 2016, 61, 35-41. [CrossRef]

11. Chatzarakis, G.E.; Džurina, J.; Jadlovská, I. A remark on oscillatory results for neutral differential equations. Appl. Math. Lett. 2019, 90, 124-130. [CrossRef]

12. Liu, S.; Zhang, Q.; Yu, Y. Oscillation of even-order half-linear functional differential equations with damping. Comput. Math. Appl. 2011, 61, 2191-2196. [CrossRef]

13. Agarwal, R.P.; Bazighifan, O.; Ragusa, M.A. Nonlinear Neutral Delay Differential Equations of Fourth-Order: Oscillation of Solutions. Entropy 2021, 23, 129. [CrossRef] [PubMed]

14. Zafer, A. Oscillation criteria for even order neutral differential equations. Appl. Math. Lett. 1998, 11, 21-25. [CrossRef]

15. Zhang, Q.; Yan, J. Oscillation behavior of even order neutral differential equations with variable coefficients. Appl. Math. Lett. 2006, 19, 1202-1206. [CrossRef]

16. Agarwal, R.; Grace, S.; O'Regan, D. Oscillation Theory for Difference and Functional Differential Equations; Kluwer Academic Publishers: Dordrecht, The Netherlands, 2000. 
17. Kiguradze, I.T.; Chanturiya, T.A. Asymptotic Properties of Solutions of Nonautonomous Ordinary Differential Equations; Kluwer Academic Publishers: Dordrecht, The Netherlands, 1993.

18. Moaaz, O.; Awrejcewicz, J.; Bazighifan, O. A new approach in the study of oscillation criteria of even-order neutral differential equations. Mathematics 2020, 8, 179. [CrossRef]

19. Agarwal, R.; Shieh, S.L.; Yeh, C.C. Oscillation criteria for second order retarde ddifferential equations. Math. Comput. Model. 1997, 26, 1-11. [CrossRef]

20. Nehari, Z. Oscillation criteria for second order linear differential equations. Trans. Am. Math. Soc. 1957, 85, 428-445. [CrossRef] 